\title{
Surface Patterning of Functional Ceramics: A Materials Design
}

\author{
Sheikh A. Akbar* \\ Department of Materials Science and Engineering, The Ohio State University, Columbus, OH, USA
}

Keywords: surface patterning, self-assembly, nanostructures, novel synthesis, large-scale patterning

\section{INTRODUCTION}

Functional ceramics can be fabricated as bulk, thick and thin film, and aggregates of inorganic compositions, often oxides. They are usually stable under extreme conditions and possess a wide range of functional properties. These materials are manufactured from affordable raw materials and are used in batteries, catalysis, fuel cells, membranes and filters, chemical sensors, bioceramics, supercapacitors, and a variety of electromagnetic and electro-optic applications. This is clearly a very diverse area that is rapidly growing, and many review articles have been written in these topics that are illuminating (Koumoto et al., 2013; Tan et al., 2013; Granqvist, 2014; Miller et al., 2014; Zai and Qian, 2015; Yu et al., 2016). It is not practical to cover all these areas with any reasonable technical depth in this short article. Instead, this focuses on a niche area of surface patterning of oxides using novel and inexpensive process pathways that do not require lithography, instead exploiting intrinsic material properties to create oriented and ordered nanostructures.

The development in science and nanotechnology in the past three decades has enabled us to design and fabricate nanoscale features on solid surfaces leading to a paradigm shift in the field of microelectronics, sensor technology, data storage, biotechnology, and semiconductor industry. While conventional applications take advantage of the size, shape, and the high specific area of the surface nanostructures, carefully designed periodic features are now being used to fabricate metamaterials with novel properties (Shalaev, 2007; Lee et al., 2012). Surface patterns can be created on solid substrates either by "top-down" approaches or by "bottom-up" methods. The top-down strategy generates patterns by imposing macroscopic controls such as masks or templates. It usually involves physical addition or removal of materials at selected regions on the substrate surface. On the other hand, one may take advantage of the intrinsic material properties to grow microscale/nanoscale surface structures and patterns-a process known as self-assembly.

Lithography is the most common top-down method for surface patterning and has been extensively used in modern microelectronic device fabrication. Conventional lithography technique, also known as photolithography, uses light and a photomask to define geometric patterns on a layer of photoresist coated on the substrate. The resolution of photolithography is generally limited by diffraction effects between the light and the mask. Higher printing resolution $(\sim 10 \mathrm{~nm})$ can be achieved by non-optical lithography techniques such as electron-beam lithography and nanoimprinting lithography. The electron-beam lithography (Vieu et al., 2000) is a maskless lithography technique where the patterns are directly drawn onto the photoresist by a focused beam of electrons. Despite its high resolution, this technique is not cost-efficient for large surface patterning due to the expensive equipment and its low throughput. Nanoimprinting lithography (Guo, 2007) is a relatively low-cost and high-throughput technology, where patterns are transferred onto the resist from nanostructured molds via imprinting. One major challenge of

nanoimprinting lithography is to fabricate durable molds with high-resolution and high-density 
nanostructures. The thermal expansion mismatch between the mold and resist is another concern since a temperature over $100^{\circ} \mathrm{C}$ is typically needed for this process. These drawbacks prompted research in alternative surface patterning techniques such as bottom-up strategies involving self-assembly.

Unlike the "top-down" methods where surface patterns are artificially created, "bottom-up" approaches rely on the intrinsic chemical properties of the materials, where surface patterns usually form spontaneously under suitable conditions via selfassembly process. Self-assembly processes can be found in a wide range of phenomena from crystallization (self-assembly of atoms) and self-assembled monolayers (self-assembly of molecules) all the way up to the formation of schools (self-assembly of fish) and the galaxies (self-assembly of planets) (Whitesides and Grzybowski, 2002). Self-assembled structures can either be directly grown on the substrates or be used as templates for etching or deposition.

Growth processes by the bottom-up approach can involve a gas-phase reaction of a vapor species and a substrate or can occur in solution. The desired end application often dictates which process is best suited for the production of nanostructures. Solution grown processes often require very precise control of the chemical properties of the solution such as $\mathrm{pH}$ and viscosity and can contain contaminate species in the precipitate precursors, which require removal once the reaction has completed. Given the limited scope of this article, solution processes are not covered and interested readers are directed to a recent review article (Lee, 2009). Vapor-assisted growth, on the other hand, has several advantages over solution methods. Foremost, the nanostructures can often be grown directly onto a desired substrate, allowing for easy integration into an existing device or product during the production process. In addition, a tight control over chemical reactants, compared to those required in solution treatments, is not typically necessary for these processes, thus allowing for both a wider margin of error in processing and a reduction in manufacturing costs.

\section{VAPOR PHASE-ASSISTED GROWTH}

Vapor phase-assisted growth is one of the most common means for producing nanostructures, often NWs, on a desired substrate. The vapor phase constituents can be a metal, as is the case with vapor-solid (VS) and vapor-liquid-solid (VLS), or it can be an oxygen-bearing gas as with growth by oxidation. Several techniques have been used to produce the vapor phase constituents needed for growth by both VS and VLS including laser ablation (Hu et al., 1999), thermal evaporation (Dailey et al., 2004), carbothermal evaporation (Wang et al., 2005; Prete et al., 2007), and metal organic precursors in the gas phase (Bao et al., 2008; Bauer et al., 2008; Soci et al., 2008).

Of the vapor phase growth methods, the VLS method is the most widely used and best understood. In general, a vaporized source material is introduced into a reaction chamber and flown by a carrier gas downstream. As this vaporized constituent deposits on a substrate surface, there must be a means of confining the deposition to limited surface locations for the process to produce nanostructures. One of the most successful ways to provide this confinement is through the use of a liquid metal catalyst, which is the basis of the VLS mechanism. A catalyst in the liquid phase, typically a metal, provides preferential deposition sites for the vaporized source material and gives the chemical adsorption confinement required for one-dimensional growth (Wagner and Ellis, 1964).

In a VS mechanism, the presence of a metal catalyst is not required for the confinement of growth to one dimension from a condensed vapor phase (Wang, 2003). Although poorly understood, the widely accepted view is that defects inherent to the substrate surface provide the growth confinement required to obtain $1 \mathrm{D}$ nanostructures. The defects can be strain induced, dislocation dependant, or impurity driven. While this method has been successfully used to produce nanostructures of $\mathrm{SnO}_{2}$ and $\mathrm{ZnO}$, this has not been successful for $\mathrm{TiO}_{2}$ because of low vapor pressure of Ti. For $\mathrm{TiO}_{2}$, other methods such as thermal oxidation and gas phase-assisted etching process have proven successful.

In the thermal oxidation process, the vapor species is oxygen rather than a vaporized metal, a distinction that separates this process from the majority of other VS methods. Whiskers and needles of cupric oxide at the nanoscale via oxidation have been reported (Kumar et al., 2004; Lin, 2004; Xu et al., 2004; Kaur et al., 2006; Fan et al., 2008). Oxidation-driven nanowire growth of $\mathrm{TiO}_{2}$ in an acetone saturated Ar environment and their applications in electro-emission and cell repellence have been reported in literature (Huo et al., 2008, 2009; Zhao et al., 2010). More recently, a simpler process has been reported (Lee et al., 2010), where rutile $\mathrm{TiO}_{2}$ nanowhiskers were grown on $\mathrm{Ti}$ and $\mathrm{Ti}$ alloys in a gas environment flowed from a commercial grade Ar cylinder containing $10 \mathrm{~s} \mathrm{ppm} \mathrm{of} \mathrm{oxygen.} \mathrm{This} \mathrm{process}$ has been successfully demonstrated on bulk, foil, thin film, and particles of commercially pure $\mathrm{Ti}$ and $\mathrm{Ti}$ alloys including Ti64 (Ti-6 wt\% Al-4 wt\% V) that is widely used as a hip implant material. Patterned surfaces by this oxidation process has lately been used in various biomedical applications (Dinan et al., 2013; Tan et al., 2014a,b, 2016).

Yet another unique process that falls under the top-down scheme is gas phase-assisted etching known as "nano-carving" (Yoo et al., 2004a,b) that produces nanofibers on well-sintered polycrystalline $\mathrm{TiO}_{2}$ exposed to a heat treatment at $700^{\circ} \mathrm{C}$ for $8 \mathrm{~h}$ in a flowing atmosphere of $5 \% \mathrm{H}_{2}-95 \% \mathrm{~N}_{2}$. The fibers form along the $<001>$ crystallographic direction of rutile $\mathrm{TiO}_{2}$ crystal and are $15-50 \mathrm{~nm}$ in diameter and typically $1 \mu \mathrm{m}$ in length. This is an anisotropic etching process where the $1 \mathrm{D}$ confinement is achieved by segregating impurities (present in the original $\mathrm{TiO}_{2}$ powder) on the surface that form protective caps. During etching, as $\mathrm{H}_{2}$ gas reacts with oxygen on the $\mathrm{TiO}_{2}$ surface forming $\mathrm{H}_{2} \mathrm{O}$ (g), Ti diffuses from the surface to the bulk filling available Ti sinks. Thus, the process stops as soon as the Ti sinks in the bulk are filled. Since breaking of Ti-O bond on the (001) surface of rutile $\mathrm{TiO}_{2}$ requires the least energy, the etching proceeds along the $<001>$ direction. These types of structures have been used for gas sensing (Yoo et al., 2004a,b) and biological cell attachment (Dinan et al., 2013). 


\section{STRESS-DRIVEN MORPHOLOGICAL INSTABILITY}

One nanoscale self-assembly method that has received significant attention is based on a stress-driven morphological instability broadly known as the Asaro-Tiller-Grinfeld (ATG) instability (Asaro and Tiller, 1972; Srolovitz, 1989). An epitaxial thin film with low lattice mismatch to its substrate is inherently unstable when it is coherently stressed. If a transport path is available, such as surface diffusion, the stored elastic energy can drive significant morphological reordering of the surface film. A competition is setup between relieving stored elastic energy and the creation of new free surface when the planar surface forms undulations or islands. To date, the most widely studied experimental systems are semiconductors, where nanostructures form during deposition as Stranski-Krastanov islands (Stranski and Krastanow, 1939; Bauer, 1958) consisting of Ge-Si solid solutions on (001) Si surfaces (Eaglesham and Cerullo, 1990; Mo et al., 1990; Cirlin et al., 1995; Ramasubramaniam and Shenoy, 2005; Vanfleet et al., 2007) with limited intrinsic capacity for alignment that persists over short distances. Thus, one must guide the pattern alignment that is mainly accomplished by creating a multilayered structure with alternating films of $\mathrm{Si}$ and $\mathrm{Ge}$ with various templates of strain modulation (Lam et al., 2002). An alternative approach is to use photolithography to introduce steps or lines at the microscale (Mo et al., 1990; Tersoff et al., 1996). The islands then self-assemble following the template set by these features. The lithographic approach, however, is limited in the type of structures and spatial order achievable. Large areas cannot be covered with precise feature control and periodicity, which can at best be achieved in 1D and much less in 2D and 3D.

Recent reports of formation of self-assembled arrays of nanostructures (Rauscher et al., 2008; Ansari et al., 2013) surrounding rare earth dopant sources on the surface of YSZ marks a new paradigm in surface patterning. The dopant sources can be put in the form of thin film or lithographically patterned patches or even in the form of powder. On annealing at approximately one-half the melting temperature of zirconia, surface diffusion of dopants leads to the breakup of the surface around the source, creating arrays of epitaxial nanoislands with a characteristic size $(\sim 100 \mathrm{~nm})$ on YSZ (001) surface and alignment along elastically compliant directions, $\langle 110\rangle$. On YSZ (110) surface, on the other hand, periodic arrays of parallel nanobars separated by channels (period $\sim 100 \mathrm{~nm}$ ) grow out of the dopant sources, covering relatively wide areas of the surface $(\sim 10 \mu \mathrm{m})$. These form via a strain-based mechanism similar to the ATG instability, whereby the stress accumulated in the doped surface of the YSZ substrate is relieved by creation of self-assembled nanostructures. Generally speaking, this type of self-assembly is similar in its underlying physical phenomena to ordering of semiconductor islands on 2D surfaces (Eaglesham and Cerullo, 1990), arrays of nickel aluminide precipitates in 3D Ni-base superalloys (Khachaturyan, 1983), and 3D semiconductor island arrays in multilayer systems (Springholz et al., 1998). Also, the ease of making these patterned substrates and the fact that the process can be scaled up to cover large surfaces make them useful master patterns for nanoimprinting to polydimethylsiloxane polymer (Zimmerman et al., 2010), which can be used directly for cell attachment and proliferation studies (Parikh et al., 2012).

Most recently, ordered surface step arrays were successfully prepared on two different types of miscut yttria-stabilized zirconia (YSZ) substrates via one-step preannealing that produced well-developed steps (Niu, 2016). These steps were subsequently used as templates for the self-assembly of the nanoislands, which resulted in significantly improved alignment of the islands along the step direction. In addition, a unique step faceting phenomenon at the island growth front was observed on the (001)-[100] miscut YSZ substrates. While the mechanism for this dopant-induced faceting has not yet been established, this opens a new avenue for surface patterning that has huge implications on adsorption, catalysis, and many other surface-reaction phenomena.

Overall, the self-assembly of both the nanoislands/nanobars and surface steps including dopant-induced faceting are promising techniques that can be used to pattern a large surface with nanoscale features. This marks a new paradigm in self-assembly literature and has multifaceted significance: (1) fabrication of ordered nanostructures in a new class of high-temperature technologically important ceramic materials; (2) simplicity of the process; the deposition can be as simple as sprinkling source materials as powders on a properly chosen substrate; and (3) exploitation of intrinsic materials properties to achieve self-assembly. In addition, a high intrinsic capacity for ordering, dictated by intrinsic material properties, is a major advantage because it implies that less tuning/guiding is needed to achieve a certain degree of order.

\section{FUTURE OUTLOOK}

The surface patterning techniques described in this article represent an innovation in nanoprocessing without requiring lithography, making them cost-effective, that will assist in the proliferation of nanodevices. These techniques are costeffective because they are (1) easy to implement, (2) inexpensive, and (3) highly scalable for mass production. Moreover, there are opportunities for multidisciplinary studies involving characterization of surface/interface structures and characteristics of gas-solid, liquid-solid interaction and biological cell attachment and proliferation on these structures. Such studies combined with computer modeling and simulation would aid in the fundamental understanding of the mechanisms allowing process control to create a wide range of nanostructures, as well as to apply the techniques to other technologically important ceramics.

In terms of broader impact, these techniques provide new and affordable process pathways for making nanostructures on bulk, thin film, and particles or even pattern transfer to other substrates by replica molding. These methods provide an economical way to mass-produce nanostructures that are attached 
to a substrate making an ideal platform for a wide variety of applications. These patterns provide high surface area and facilitate studies of a variety of applications that require interaction on the surface such as chemical sensing (Miller et al., 2014), superwetting surface fabrication (Feng and Jiang, 2006), and biological cell attachment and proliferation (Tan et al., 2013). Further improved alignment and periodicity of these nanostructures should yield new applications in other areas such as metamaterial fabrication (Shalaev, 2007), field enhancement by light-material interaction (Kim et al., 2008), and nanodevice fabrication (Carlson et al., 2012).

\section{REFERENCES}

Ansari, H. M., Dixit, V., Zimmerman, L. B., Rauscher, M. D., Dregia, S. A., and Akbar, S. A. (2013). Self assembly of nanoislands on YSZ-(001) surface: a mechanistic approach toward a robust process. Nano Lett. 13, 2116-2121. doi:10.1021/nl4005282

Asaro, R., and Tiller, W. (1972). Interface morphology development during stress corrosion cracking: part I. via surface diffusion. Metall. Mater. Trans. B 3, 1789-1796. doi:10.1007/BF02642562

Bao, X. Y., Soci, C., Susac, D., Bratvold, J., Aplin, D. P. R., Wei, W., et al. (2008). Heteroepitaxial growth of vertical GaAs nanowires on $\mathrm{Si}$ (111) substrates by metal-organic chemical vapor deposition. Nano Lett. 8, 3755-3760. doi:10.1021/ nl802062y

Bauer, E. (1958). Phanomenologische Theorie Der Kristallabscheidung An Oberflachen. II. Zeitschrift Fur Kristallographie 110, 395-431. doi:10.1524/ zkri.1958.110.1-6.395

Bauer, J., Gottschalch, V., and Wagner, G. (2008). The influence of the droplet composition on the vapor-liquid-solid growth of InAs nanowires on GaAs (111)[sub B] by metal-organic vapor phase epitaxy. J. Appl. Phys. 104, 114315. doi:10.1063/1.3033556

Carlson, A., Bowen, A. M., Huang, Y., Nuzzo, R. G., and Rogers, J. A. (2012). Transfer printing techniques for materials assembly and micro/nanodevice fabrication. Adv. Mater. 24, 5284-5318. doi:10.1002/adma.201201386

Cirlin, G. E., Guryanov, G. M., Golubok, A. O., Tipissev, S. Ya., Ledentsov, N. N., Kop'ev, P. S., et al. (1995). Ordering phenomena in InAs strained layer morphological transformation on GaAs (100) surface. Appl. Phys. Lett. 67, 97. doi:10.1063/1.115520

Dailey, J. W., Taraci, J., Clement, I., Smith, D. J., Drucker, J., and Picraux, S. T. (2004). Vapor-liquid-solid growth of germanium nanostructures on silicon. J. Appl. Phys. 96, 7556-7567. doi:10.1063/1.1815051

Dinan, B., Gallego-Perez, D., Lee, H., Hansford, D., and Akbar, S. A. (2013). Thermally grown $\mathrm{TiO}_{2}$ nanowires to improve cell growth and proliferation on titanium based materials. Ceram. Int. 39, 5949-5954. doi:10.1016/ j.ceramint.2012.12.004

Eaglesham, D., and Cerullo, M. (1990). Dislocation-free Stranski-Krastanow growth of Ge on Si (100). Phys. Rev. Lett. 64, 1943-1946. doi:10.1103/ PhysRevLett.64.1943

Fan, X. Y., Wu, Z. G., Yan, P. X., Geng, B. S., Li, H. J., Li, C., et al. (2008). Fabrication of well-ordered $\mathrm{CuO}$ nanowire arrays by direct oxidation of sputter-deposited $\mathrm{Cu}_{3} \mathrm{~N}$ film. Mater. Lett. 62, 1805-1808. doi:10.1016/j.matlet.2007.10.006

Feng, X., and Jiang, L. (2006). Design and creation of superwetting/antiwetting surfaces. Adv. Mater. 18, 3063-3078. doi:10.1002/adma.200501961

Granqvist, C. G. (2014). Electrochromics for smart windows: oxide-based thin films and devices. Thin Solid Films 564, 1-38. doi:10.1016/j.tsf.2014.02.002

Guo, L. J. (2007). Nanoimprint lithography: methods and material requirements. Adv. Mater. 19, 495-513. doi:10.1002/adma.200600882

Hu, J., Odom, T. W., and Lieber, C. M. (1999). Chemistry and physics in one dimension: synthesis and properties of nanowires and nanotubes. Acc. Chem. Res. 435-445. doi:10.1021/ar9700365

Huo, K., Zhang, X., Fu, J., Qian, G., Xin, Y., Zhu, B., et al. (2009). Synthesis and field emission properties of rutile $\mathrm{TiO}_{2}$ nanowires arrays grown directly on a $\mathrm{Ti}$ metal self-source substrate. J. Nanosci. Nanotechnol. 9, 3341-3346. doi:10.1166/ jnn.2009.VC09

\section{AUTHOR CONTRIBUTIONS}

The author confirms being the sole contributor of this work and approved it for publication.

\section{ACKNOWLEDGMENTS}

This article is written based on Ph.D. dissertations of my former students, Drs. Sehoon Yoo, Michael Rausher, Huyong Lee, Benjamin Dinan, Haris Ansari, and Zhiyuan Niu. Professor Dregia co-advised many of these students.

Huo, K., Zhang, X., Hu, L., Sun, X., Fu, J., and Chu, P. K. (2008). One-step growth and field emission properties of quasialigned $\mathrm{TiO}$ [sub 2] nanowire/carbon nanocone core-shell nanostructure arrays on Ti substrates. Appl. Phys. Lett. 93, 013105. doi:10.1063/1.2955519

Kaur, M., Muthe, K. P., Despande, S. K., Choudhury, S., Singh, J. B., Verma, N., et al. (2006). Growth and branching of $\mathrm{CuO}$ nanowires by thermal oxidation of copper. J. Cryst. Growth 289, 670-675. doi:10.1016/j.jcrysgro.2005.11.111

Khachaturyan, A. G. (1983). "Theory of structural transformations in solids," in Materials Research Bulletin. New York: John Wiley \& Sons. doi:10.1016/0025-5408(84)90018-7

Kim, S., Jin, J., Kim, Y. J., Park, I. Y., Kim, Y., and Kim, S. W. (2008). High-harmonic generation by resonant plasmon field enhancement. Nature 453, 757-760. doi:10.1038/nature07012

Koumoto, K., Funahashi, R., Guilmeau, E., Miyazaki, Y., Weidenkaff, A., Wang, Y., et al. (2013). Thermoelectric ceramics for energy harvesting. J. Am. Ceram. Soc. 96, 1-23. doi:10.1111/jace. 12076

Kumar, A., Srivastava, A. K., Tiwari, P., and Nandedkar, R. V. (2004). The effect of growth parameters on the aspect ratio and number density of $\mathrm{CuO}$ nanorods. J. Phys. Condens. Matter 16, 8531-8543. doi:10.1088/0953-8984/16/47/007

Lam, C.-H., Lee, C.-K., and Sander, L. M. (2002). Competing roughening mechanisms in strained heteroepitaxy: a fast kinetic Monte Carlo Study. Phys. Rev. Lett. 89, 216102. doi:10.1103/PhysRevLett.89.216102

Lee, H., Dregia, S., Akbar, S., and Alhoshan, M. (2010). Growth of 1-D $\mathrm{TiO}_{2}$ nanowires on $\mathrm{Ti}$ and $\mathrm{Ti}$ alloys by oxidation. J. Nanomater. 2010, 1-7. doi:10.1155/2010/503186

Lee, J.-H. (2009). Gas sensors using hierarchical and hollow oxide nanostructures: overview. Sens. Actuat. B Chem. 140, 319-336. doi:10.1016/j.snb.2009.04.026

Lee, J.-H., Singer, J. P., and Thomas, E. L. (2012). Micro-/nanostructured mechanical metamaterials. Adv. Mater. 24, 4782-4810. doi:10.1002/adma. 201201644

Lin, H.-H. (2004). Characterizing well-ordered $\mathrm{CuO}$ nanofibrils synthesized through gas-solid reactions. J. Appl. Phys. 95, 5889. doi:10.1063/1.1690114

Miller, D. R., Akbar, S. A., and Morris, P. A. (2014). Nanoscale metal oxide-based heterojunctions for gas sensing: a review. Sens. Actuat. B Chem. 204, 250-272. doi:10.1016/j.snb.2014.07.074

Mo, Y. W., Savage, D. E., Swartzentruber, B. S., and Lagally, M. G. (1990). Kinetic pathway in Stranski-Krastanov growth of $\mathrm{Ge}$ on $\mathrm{Si}(001)$. Phys. Rev. Lett. 65, 1020-1023. doi:10.1103/PhysRevLett.65.1020

Niu, Z. (2016). Nanoscale Surface Patterning and Engineering of YSZ Surfaces. Ph.D. dissertation, The Ohio State University, Columbus, OH.

Parikh, K. S., Rao, S. S., Ansari, H. M., Zimmerman, L. B., Lee, L. J., Akbar, S. A., et al. (2012). Ceramic nanopatterned surfaces to explore the effects of nanotopography on cell attachment. Mater. Sci. Eng. C 32, 2469-2475. doi:10.1016/j. msec.2012.07.028

Prete, P., Lovergine, N., and Tapfer, L. (2007). Nanostructure size evolution during $\mathrm{Au}$-catalysed growth by carbo-thermal evaporation of well-aligned $\mathrm{ZnO}$ nanowires on (100)Si. Appl. Phys. A 88, 21-26. doi:10.1007/s00339-007-3946-4

Ramasubramaniam, A., and Shenoy, V. B. (2005). Growth and ordering of Si-Ge quantum dots on strain patterned substrates. J. Eng. Mater. Technol. 127, 434. doi:10.1115/1.1924559

Rauscher, M. D., Boyne, A., Dregia, S. A., and Akbar, S. A. (2008). Self-assembly of pseudoperiodic arrays of nanoislands on YSZ-(001). Adv. Mater. 20, 1699-1705. doi:10.1002/adma.200701383 
Shalaev, V. M. (2007). Optical negative-index metamaterials. Nat. Photonics 1, 41-48. doi:10.1038/nphoton.2006.49

Soci, C., Bao, X. Y., Aplin, D. P. R., and Wang, D. L. (2008). A systematic study on the growth of GaAs nanowires by metal-organic chemical vapor deposition. Nano Lett. 8, 4275-4282. doi:10.1021/N1801986r

Springholz, G., Holy, V., Pinczolits, M., and Bauer, C. (1998). Self-organized growth of three-dimensional quantum-dot cyrstals with Fcc-like stacking and a tunable lattice constant. Science 282, 734-737. doi:10.1126/science.282.5389.734

Srolovitz, D. J. (1989). On the stability of surfaces of stressed solids. Acta Metall. 37, 621-625. doi:10.1016/0001-6160(89)90246-0

Stranski, I. N., and Krastanow, L. (1939). Berichtigung Zur Arbeit - 'Zur Theorie Der Orientierten Ausscheidung von Ionenkristallen Aufeinander'. Monatshefte Fur Chemie 72, 76. doi:10.1007/BF02716117

Tan, A. W., Dalilottojari, A., Pingguan-Murphy, B., Ahmad, R., and Akbar, S. (2014a). In vitro chondrocyte interactions with $\mathrm{TiO}_{2}$ nanofibers grown on Ti-6Al-4V substrate by oxidation. Ceram. Int. 40, 8301-8304. doi:10.1016/ j.ceramint.2014.01.032

Tan, A. W., Ismail, R., Chua, K. H., Ahmad, R., Akbar, S. A., and Pingguan-Murphy, B. (2014b). Osteogenic potential of in situ $\mathrm{TiO}_{2}$ nanowire surfaces formed by thermal oxidation of titanium alloy substrate. Appl. Surf. Sci. 320, 161-170. doi:10.1016/j.apsusc.2014.08.160

Tan, A. W., Liau, L. L., Chua, K. H., Ahmad, R., Akbar, S. A., and Pingguan-Murphy, B. (2016). Enhanced in vitro angiogenic behaviour of human umbilical vein endothelial cells on thermally oxidized $\mathrm{TiO}_{2}$ nanofibrous surfaces. Sci. Rep. 6 , 21828. doi:10.1038/srep21828

Tan, A. W., Pingguan-Murphy, B., Ahmad, R., and Akbar, S. A. (2013). Advances in fabrication of $\mathrm{TiO}_{2}$ nanofiber/nanowire arrays toward the cellular response in biomedical implantations: a review. J. Mater. Sci. 48, 8337-8353. doi:10.1007/ s10853-013-7659-0

Tersoff, J., Teichert, C., and Lagally, M. G. (1996). Self-organization in growth of quantum dot superlattices. Phys. Rev. Lett. 76, 1675-1678. doi:10.1103/ PhysRevLett.76.1675

Vanfleet, R. R., Basile, D. P., Kamins, T. I., Silcox, J., and Williams, R. S. (2007). Silicon-germanium interdiffusion and interfaces in self-assembled quantum dots. Appl. Phys. A Mater. Sci. Process. 86, 1-9. doi:10.1007/s00339-006-3724-8

Vieu, C., Carcenac, F., Pepin, A., Chen, Y., Mejias, M., Lebib, A., et al. (2000). Electron beam lithography - resolution limits and applications. Appl. Surf. Sci. 164, 111-117. doi:10.1016/0167-9317(95)00368-1

Wagner, R. S., and Ellis, W. C. (1964). Applied physics letters 1. Appl. Phys. Lett. 4, 89-90. doi:10.1063/1.1753975
Wang, B., Yang, Y. H., Wang, C. X., and Yang, G. W. (2005). Growth and photoluminescence of $\mathrm{SnO}_{2}$ nanostructures synthesized by Au-Ag alloying catalyst assisted carbothermal evaporation. Chem. Phys. Lett. 407, 347-353. doi:10.1016/j.cplett.2005.03.119

Wang, Z. L. (2003). Nanobelts, nanowires, and nanodiskettes of semiconducting oxides - From materials to nanodevices. Adv. Mater. 15, 432-436. doi:10.1002/ adma. 200390100

Whitesides, G. M., and Grzybowski, B. (2002). Self-assembly at all scales. Science 295, 2418-2421. doi:10.1126/science.1070821

$\mathrm{Xu}, \mathrm{C} . \mathrm{H}$., Woo, C. H., and Shi, S. Q. (2004). Formation of $\mathrm{CuO}$ nanowires on $\mathrm{Cu}$ foil. Chem. Phys. Lett. 399, 62-66. doi:10.1016/j.cplett.2004.10.005

Yoo, S., Akbar, S. A., and Sandhage, K. H. (2004a). Nanocarving of bulk titania crystals into oriented arrays of single-crystal nanofibers via reaction with hydrogen-bearing gas. Adv. Mater. 16, 260-264. doi:10.1002/adma. 200305781

Yoo, S., Akbar, S. A., and Sandhage, K. H. (2004b). Nanocarving of titania $\left(\mathrm{TiO}_{2}\right)$ : a novel approach for fabricating chemical sensing platform. Ceram. Int. 30, 1121-1126. doi:10.1016/j.ceramint.2003.12.085

Yu, X., Marks, T. J., and Facchetti, A. (2016). Metal oxides for optoelectronic applications. Nat. Mater. 15, 383-396. doi:10.1038/nmat4599

Zai, J., and Qian, X. (2015). Three dimensional metal oxides-graphene composites and their applications in lithium ion batteries. RSC Adv. 5, 8814-8834. doi:10.1039/C4RA11903G

Zhao, L., Hu, L., Huo, K., Zhang, Y., Wu, Z., and Chu, P. K. (2010). Mechanism of cell repellence on quasi-aligned nanowire arrays on Ti alloy. Biomaterials 31, 8341-8349. doi:10.1016/j.biomaterials.2010.07.036

Zimmerman, L. B., Rauscher, M. D., Ellis, J., Boukany, P., and Lee, L. J. (2010). Nanoimprinting using self-assembled ceramic nanoislands. Nanotechnology 21, 045304. doi:10.1088/0957-4484/21/4/045304

Conflict of Interest Statement: The author declares that the research was conducted in the absence of any commercial and financial relationships that could be construed as a potential conflict of interest.

Copyright (C) 2017 Akbar. This is an open-access article distributed under the terms of the Creative Commons Attribution License (CC BY). The use, distribution or reproduction in other forums is permitted, provided the original author(s) or licensor are credited and that the original publication in this journal is cited, in accordance with accepted academic practice. No use, distribution or reproduction is permitted which does not comply with these terms. 